\title{
MULTIPLE FREQUENCY EPR STUDIES ON THREE FORMS OF OXIDIZED CYTOCHROME $c$ OXIDASE
}

\author{
WILLIAM R. DUNHAM ${ }^{\mathrm{a}, *}$, RICHARD H. SANDS ${ }^{a}$, ROBERT W. SHAW ${ }^{\mathrm{b}, * *}$ and HELMUT BEINERT ${ }^{\mathrm{d}}$ \\ ${ }^{a}$ Biophysics Research Division, Institute of Science and Technology, The University of Michigan, Ann Arbor, MI 48109, and ${ }^{b}$ Institute for \\ Enzyme Research, University of Wisconsin, Madison, WI 53706 (U.S.A.)
}

(Received March 30th, 1983)

(Revised manuscript received July 7 th, 1983)

Key words: ESR; Cytochrome c oxidase; Spin-coupling; (Bovine heart mitochondria)

Bovine heart mitochondrial cytochrome $c$ oxidase (cytochrome $a a_{3}$ ) (EC 1.9.3.1) has been demonstrated to occur in several forms when the redox centers in the protein are thought to be fully oxidized. We report here the results of extensive EPR studies at 3, 8.9, 9.2, 9.4, 15 and $34 \mathrm{GHz}$ on the resting state, the alternative resting state (with $g=12$ at $9 \mathrm{GHz}$ ) and pulsed state (with $g=5$ signal at $9 \mathrm{GHz}$ ). Theoretical consideration is given to all binary spin-coupling possibilities under the constraint that the iron atoms are either ferric or ferrous and the copper atoms are either cupric or cuprous. We conclude that the $g=12$ signal can arise from any spin system with $S>1$ and $|D|=0.15 \mathrm{~cm}^{-1}$. The $g=5$ signals originate from an excited, integer-spin system with $|D|=0.035 \mathrm{~cm}^{-1}$, which is approximately $7 \mathrm{~cm}^{-1}$ above the ground state (not observed in EPR). It is pointed out that in interpretations of data and elaboration of suitable models in this field, the implications of spin-coupling should be considered in a comprehensive and not in a selective way. At 3 GHz, EPR spectra of $\mathrm{Cu}_{\mathrm{A}}$ in the resting, pulsed and anaerobically oxidized states show that this center is identical in its EPR for all three states.

\section{Introduction}

In recent years cytochrome $c$ oxidase (EC 1.9.3.1) has been intensively studied from a variety of viewpoints. Many advanced physical techniques have been used in attempts to define the state and relationships of the four metal centers in the enzyme. The current picture is that these metal centers can occur in a number of states, which are determined not only by the number of electrons taken up from substrate - as one would expect - but also by conformational responses of the protein to substrates, which may take place on

\footnotetext{
* To whom correspondence should be sent.

** Present address: Chemistry Department, Texas Tech University, Lubbock, TX 79409, U.S.A.
}

widely differing time-scales. Such responses may also be evoked by non-substrate ligands or other external influences and will modulate the relationships between the metal centers present.

A promising approach toward simplifying the ensuing complicated situation has been the attempt to correlate the results of the more discriminating physical methods and, on that basis, narrow down the number of possibilities as to the nature of the states to those that are compatible with all of the results [1-5]. This paper respresents a contribution to such efforts. We present EPR measurements, calculations and considerations on three states of oxidized cytochrome oxidase, viz., the resting state, the alternative resting state showing the so-called $g=12$ EPR signal and transient 'pulsed' [6] state exhibiting the $g=5$ signal $[7,8]$. The principal experimental tool was EPR spectros- 
copy at different microwave frequencies and the evaluation was largely patterned after that used by others $[9,10]$. This work was presented in part at the Third Symposium on Oxidases and Related Oxidation-reduction Systems at Albany, NY, in 1979 [11].

\section{Materials and Methods}

Sources of materials, biochemical approaches and EPR spectroscopy at 9 and $34 \mathrm{GHz}$ have been described in our previous publications $[8,11-13]$. The total heme concentration was between 0.5 and $1 \mathrm{mM}$ in all experiments. Details on specific experiments will be found in the legends to the appropriate figures. All EPR spectra were taken under conditions where no saturation or modulation broadening occurred. The S-band spectrometer was constructed at Ann Arbor by Strong [14] and subsequently modified by Stevenson [15]. It has a quartz-filled, dielectric cavity operating in the TE-102 mode. The bridge is a standard reflection type with a Sperry $2 \mathrm{~K} 41$ klystron as an energy source and a Microwave Associates MA70074 diode as a detector. The P-band Spectrometer was also constructed in Ann Arbor by Reid [16] and subsequently modified by Stevenson [15]. It has a cylindrical cavity, operating in the TE011 mode, whose resonant frequency is adjustable by moving the cavity end-walls. The tunable cavity was necessitated by the extremely narrow frequency range of the klystron, a Varian VA94B. Details of the electronics and cryogenics are available in the theses of the above-mentioned associates. The multi-frequency, X-band EPR was done in a cavity (rectangular TE102), modified to accept styrene and sapphire rods at its E-field maximum. By shortening the cavity so that it resonated at 9.4 $\mathrm{GHz}$ without dielectric rods, we could obtain 9.2 $\mathrm{GHz}$ with the styrene and $8.9 \mathrm{GHz}$ with the sapphire rods.

\section{Theory}

The interaction between two spin systems, $S_{1}$ and $S_{2}$, can be electrostatic (exchange) or magnetic (dipole-dipole). In any case, it can be described mathematically as $\vec{S}_{1} \cdot \tilde{J} \cdot \vec{S}_{2}$ where $\tilde{J}$ is a $3 \times 3$ Cartesian tensor [17]. The tensor, $\tilde{J}$, has nine de- grees of freedom associated with the representation $D_{1} \times D_{1}$. This representation can be reduced to the sum of three irreducible representations $\boldsymbol{D}_{0}$, $D_{1}$ and $D_{2}$. Alternatively, one can write the elements of $\tilde{J}$ as a matrix $J_{i j}$. This matrix can be written as the sum of three terms as follows:

$J_{i j}=\delta_{i j} \operatorname{tr}(J) / 3+\left\{\left(J_{i j}+J_{i j}\right) / 2-\delta_{i j} \operatorname{tr}(J) / 3\right\}+\left\{J_{i j}-J_{i j}\right\} / 2$

The first term is a scalar (represented in $D_{0}$ ) and is the isotropic exchange interaction whose Hamiltonian is often written as

$\mathscr{K}=-2 J \vec{S}_{1} \cdot \vec{S}_{2}$

This interaction gives rise to spin-coupling, whereby $\vec{S}_{1}+\vec{S}_{2}=\vec{S}$. The spin-coupling also creates the possibility that one can transform the basis set of the Hamiltonian operator to advantage. For example, a convenient choice of basis set for operators with $S_{1}$ and $S_{2}$ terms is the product basis set, $\left|S_{1} m_{1}\right\rangle\left|S_{2} m_{2}\right\rangle$. With large spin-coupling terms in the Hamiltonian, one can choose, alternatively, a basis set, $\left|S_{1} S_{2} S m\right\rangle$, where $S_{1}+S_{2}=S$. In this basis set, the resultant spin, $S$, is quantized along the $z$-axis so that its projection, $m$, is a good quantum number, whereas $m_{1}$ and $m_{2}$ are not good quantum numbers. In the product basis set, both $m_{1}$ and $m_{2}$ are good quantum numbers, but $S$ and $m$ are not. The possible values for $S$ are restricted by the following relationship:

$\left|S_{1}-S_{2}\right|<S<S_{1}+S_{2}$

One can transform a calculation between the product and spin-coupled basis sets by using vectorcoupling coefficients [18]. Of course, the final solution to an eigensystem does not depend on the choice of basis set for a calculation; however, the initial operator matrix may take on a more diagonal form depending on the choice of basis set. This is the case for the isotropic exchange interaction if the spin-coupled basis set is chosen. In the spincoupled basis system, one can rewrite the isotropic exchange interaction as:

$\mathscr{K}=J\left\{S_{1}\left(S_{1}+1\right)+S_{2}\left(S_{2}+1\right)-S(S+1)\right\}$

Thus, the interaction is only on the diagonal of the 
Hamiltonian operator matrix and has a form which is easily evaluated. This matrix has the dimension $\left(2 S_{1}+1\right)\left(2 S_{2}+1\right)$. By inspection of Eqn. 1, one can see that, given values for $S_{1}, S_{2}$ and $S$, the states $\mid S_{1} S_{2} S m>$ will have the same eigenvalue for all values of $m$. Therefore, the result of the isotropic exchange interaction is the formation of multiplets denoted by the quantum number, $S$, each with a degeneracy equal to $2 S+1$.

The third term in Eqn. 1 describes a traceless, anti-symmetric tensor. The tensor has three degrees of freedom and is represented in the $D_{1}$ representation of the rotation group. The Hamiltonian for this interaction can therefore be written as

$$
\mathscr{K}_{\mathrm{a}}=\vec{C} \cdot \vec{S}_{1} \times \vec{S}_{2}
$$

where the $C$ vector is the same as the $D$ vector in the work of Moriya [19]. Since $C$ is assumed to be mainly the result of spin-orbit interactions, Moriya estimates its value at

$$
C=J(\Delta g / g)
$$

where $\Delta g$ is the $g$-shift due to the spin-orbit interaction. In terms of the spin-coupled basis set, it should be noted that the $S$-multiplets formed by the isotropic exchange interaction are mixed by the operator, $\vec{S}_{1} \times \vec{S}_{2}$, and that there are no elements of this operator within the multiplet submatrices. Therefore, the effect of $\mathcal{H}_{\mathrm{a}}$ on the eigenvalues and vectors is much less than one would assume by the size of its elements. In fact, the presence of this term has only been observed to be the cause of 'weak ferromagnetism' in crystals that are predominantly antiferromagnetic [20] and has recently been demonstrated for pairs of $\mathrm{O}_{2}^{-}$ions in potassium iodide [21].

The second term in Eqn. 1 is a symmetric matrix and has five degrees of freedom, represented in $\boldsymbol{D}_{2}$. For example, we can write this term as

$$
\begin{aligned}
\mathscr{K}_{\mathrm{s}}= & R^{\dagger}(\alpha, \beta, \gamma) \cdot D\left\{S_{z}^{2}-S(S+1) / 3+\eta\left(S_{x}^{2}+S_{y}^{2}\right)\right\} \\
& \times R(\alpha, \beta, \gamma)
\end{aligned}
$$

where the five degrees of freedom are $D, \eta$ and the three Euler angles: $\alpha, \beta$ and $\gamma$. The matrix, $R$, is the representation for Euler rotation which is ap- propriate to the basis set and particular convention for Euler rotation definition: ie., Whitaker or Goldstein convention [22]. From the form of the operator in Eqn. 7, one can see that the elements of this part of the spin-Hamiltonian will lie within spin multiplets in $S$. Thus, in the spin-coupled basis set, the operator matrix for Eqn. 7 will have a block diagonal form where each block has the dimension $2 S+1$, and where $S$ denotes the resultant spin appropriate to the block. This will be true for any values of $\alpha, \beta$ and $\gamma$, since rotation can only transform the elements of a $D_{2}$ representation within the particular sub-manifold. Thus, the general form of Eqn. 7 can have the two terms shown in Eqn. 7 plus the other three terms in $S_{x} S_{y}$, $S_{y} S_{z}$ and $S_{x} S_{z}$; all of which will have non-zero elements only within an $S$-multiplet. Therefore, the symmetric part of the $J$-tensor can do nothing to break down the spin-coupling although it will given rise to fine structure within the $S$-multiplets. This symmetric part of the exchange interaction Hamiltonian matrix can have three main contributions: (1) from the exchange interaction itself, (2) from zero-field splitting terms arising from the contributing spins, $S_{1}$ and $S_{2}$, and (3) from the magnetic dipole-dipole interaction.

The exchange contribution to the term in Eqn. 7 arises from the spin-orbit interaction. Therefore, this contribution can be treated analogously to the $g$-tensor and one can make the argument [19] that

$D / 3 \simeq J(\Delta g / g)^{2}$

where $D$ and $J$ are defined above and $\Delta g$ is the deviation of the $g$-tensor from its scalar value, $g$, in the absence of spin-orbit interaction.

Zero-field splitting terms in the spin Hamiltonians of the interacting spin systems, for example

$D_{1}\left\{S_{1 z}^{2}-S_{1}\left(S_{1}+1\right) / 3\right\}$ or $D_{2}\left\{S_{2 z}^{2}-S_{2}\left(S_{2}+1\right) / 3\right\}$

will have non-zero 'projections' into the $S$-multiplets. In other words, they will give rise to terms that lie in Eqn. 7. The calculation of these projected terms is straightforward and examples are given later in this paper and elsewhere [11,23].

The dipole-dipole interaction takes the form

$\mathcal{K}_{\mathrm{d}}=\vec{\mu}_{1} \cdot \vec{\mu}_{2} / r^{3}-3\left(\vec{\mu}_{1} \cdot \hat{r}\right)\left(\vec{\mu}_{2} \cdot \hat{r}\right) / r^{5}$ 
if $\vec{\mu}_{1}=\beta \tilde{G}_{1} \cdot \vec{S}_{1}$ and $\vec{\mu}_{2}=\beta \tilde{G}_{2} \cdot \vec{S}_{2}$ where $\tilde{G}_{1}$ and $\tilde{G}_{2}$ are the $g$-tensors for their respective spin systems, then the dipole-dipole Hamiltonian can be written as

$\mathcal{K}_{\mathrm{d}}=\beta^{2} \vec{S}_{1}^{\dagger} \cdot \tilde{T}_{12} \cdot \vec{S}_{2} / r^{3}$

where $\tilde{T}$ is a tensor given by

$\tilde{T}_{12}=\tilde{G}_{1}^{\dagger} \tilde{G}_{2}-3 \tilde{G}_{1}^{\dagger} \hat{r} \hat{f}^{\dagger} \tilde{G}_{2}$

where $\hat{r}$ is the unit vector along the center-to-center direction for the two interacting spin systems. Since $\hat{T}$ is a tensor it can be decomposed as shown in Eqn. 1 to

$$
\begin{aligned}
T_{12}= & \operatorname{tr}\left(\tilde{T}_{12}\right) / 3 \cdot 1+\left\{\left(\tilde{T}_{12}+\tilde{T}_{21}\right) / 2-\operatorname{tr}\left(\tilde{T}_{12}\right) / 3 \cdot 1\right\} \\
& +\left\{\tilde{T}_{12}-\tilde{T}_{21}\right\} / 2
\end{aligned}
$$

The three tensors on the right-hand side of this equation are difficult to evaluate for arbitrary $g$-tensors. However, by assuming that $\tilde{G}_{1}=g_{1} \cdot 1$ and $\tilde{G}_{2}=g_{2} \cdot 1$, the tensor can be evaluated as

$\tilde{T}_{12}=g_{1} g_{2}\left(1-3 \hat{p} \hat{r}^{\dagger}\right)$

a traceless, symmetric tensor, which by analogy to the above arguments for Eqn. 7 is shown to be equivalent to a contribution to the term $\mathcal{F}_{\mathrm{s}}$. The scalar and antisymmetric terms from the dipole-dipole interaction contribute the $J$ and $C$, respectively, but are on the order of the $g$-tensor asymmetries times the dipolar energy and are therefore negligible.

The Hamiltonian,

$\mathscr{K}_{z}=g \beta H_{z} S_{z}+D\left\{S_{z}^{2}-S(S+1) / 3+\eta\left(S_{x}^{2}-S_{y}^{2}\right)\right\}$

has been discussed previously [11,23] as it applies to the EPR spectra of cytochrome $c$ oxidase. We do not intend to repeat the discussions of these papers, but will make some general remarks about the Hamiltonian.

From the above discussion, it should be clear that this is not the most general Hamiltonian that can be assumed for high-spin systems. We have purposely ignored higher-order terms in the spin operator and explicit effects due to spin-orbit coupling. We are currently investigating the effects of some of these terms; however, at present, we shall only deal with the above form for the zero-field splitting. There are several coordinate systems implicit in the Hamiltonian. The zero-field splitting part of this Hamiltonian (the part proportional to $D$ ) is specified in the crystal coordinate system. This operator is represented in the group, $D_{2}$, and has five degrees of freedom in its most general form. Two of these degrees of freedom are shown in Eqn. 15 as the parameters $D$ and $\eta$ (sometimes calculations have $E=\eta D$ ). The other three degrees of freedom are the angles which specify the relationship between the laboratory and crystal frames via an Euler rotation. In Eqn. 15, these angles are absent; therefore, the Euler angles must all be zero; the subscript on the left side of the equation refers to crystal coordinates. The subscripts on the right side refer to laboratory frame coordinates.

To illustrate this point, we can rotate the crystal frame by ninety degrees about the $y$-axis. This rotation is equivalent mathematically to switching $S_{x}^{2}$ and $S_{z}^{2}$. The Hamiltonian then becomes

$\mathscr{K}_{x}=g \beta H_{z} S_{z}+D\left\{S_{x}^{2}-S(S+1) / 3+\eta\left(S_{z}^{2}-S_{y}^{2}\right)\right\}$

which can be rearranged into spherical harmonics as

$$
\begin{aligned}
\mathscr{H}_{x}= & g \beta H_{z} S_{z}+D\left\{(-1 / 2+3 \eta / 2)\left[S_{z}^{2}-S(S+1) / 3\right]\right. \\
& \left.+(1+\eta)\left(S_{x}^{2}-S_{y}^{2}\right) / 2\right\}
\end{aligned}
$$

and likewise in the $y$-direction:

$$
\begin{aligned}
\mathcal{K}_{y}= & g \beta H_{z} S_{z}+D\left\{(-1 / 2-3 \eta / 2)\left[S_{z}^{2}-S(S+1) / 3\right]\right. \\
& \left.+(\eta-1)\left(S_{x}^{2}-S_{y}^{2}\right) / 2\right\}
\end{aligned}
$$

Eqns. 15, 17 and 18 are the same Hamiltonian, for the crystal orientations along the $x, y, z$ axes. Along these three directions, the zero-field portion is decomposed into a form with non-zero coefficients of only two of the five degrees of freedom in $D_{2}$. Along any other direction, the coefficients of the other three will be non-zero. This fact has important mathematical consequences because it implies that the form of the Hamiltonian matrix is more complicated 'off-axis' than it is along the three principal directions of the crystal frame. 
Along with this complication comes a factor of eight in computer time required to solve the eigensystem.

For non-integer spin systems $(S=3 / 2,5 / 2$, ...) at zero-field, the Hamiltonian blocks into two identical matrices. The solutions to these matrices are, thus, two-fold degenerate and are called Kramer's doublets. With non-zero applied magnetic field, this degeneracy is removed. It is important to realize that even for small fields ( $>20$ $\mathrm{G}$, the largest estimated magnetic hyperfine field), the full projection of the Kramer's states is developed. If we define the EPR resonance condition as $h \nu=g^{\prime} \beta H$, then $g^{\prime}$, the spectroscopic splitting parameter, can be used to describe a resonance position. For non-integer spin systems, the resonances show a well-defined $g^{\prime}$, independent of microwave of microwave frequency, as long as $h \nu$ is much smaller than $D$. We contrast this situation to that for integer spin systems where the zero-field states are non-degenerate, diamagnetic and do not exhibit well-defined $g^{\prime}$-values until $h \nu$ is much greater than $D$. If we assume that the $g$-tensor is symmetric, then there are eight degrees of freedom in the zero-field splitting plus electron Zeeman Hamiltonian: three in the principal axis values of the $g$-tensor plus five in the zero-field splitting tensor. In order to simulate an EPR spectrum, the Hamiltonian matrix must be diagonalized interatively in order to meet the resonance condition, or, alternatively, the Hamiltonian can be solved at every field in the spectrum for each orientation. Computer programs to simulate these EPR signals are very complicated and expensive to run for spin systems greater than $S=1$. Instead, we have chosen to calculate only the resonances along the three principal axes of the crystal frame where, as mentioned above, the calculation is greatly simplified due to a blocking and subsequent reduction of the rank of the Hamiltonian matrix which must be solved. Another consequence of our choice not to look at the 'off-axis' resonances is that none of the so-called 'half-field' resonances will be represented in our diagrams. The strength of these

Fig. 1. Positions of the EPR resonances along the principal axis directions of a $S=2$ spin system with $\eta=0.07:$ A, magnetic field along the $x$-axis; B, magnetic field along the $y$-axis; C, magnetic field along the $z$-axis.
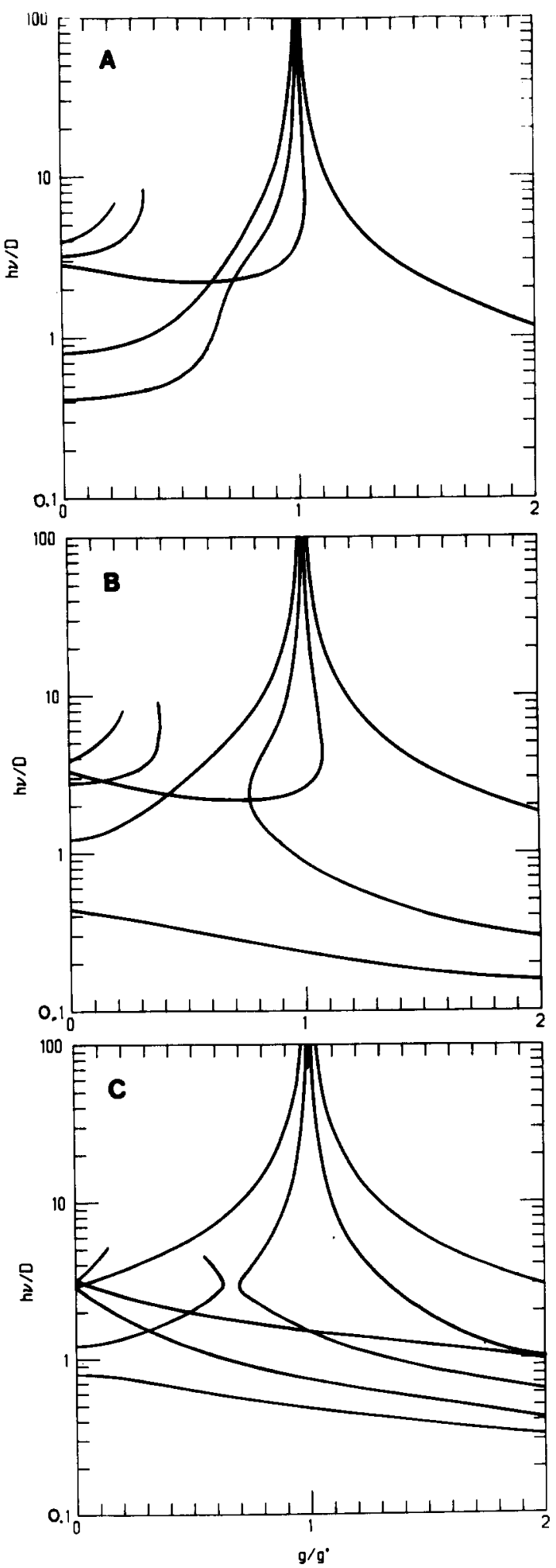
resonances is a complicated matter which we have chosen to ignore here, but which is well-described by Schepler [24] for two weakly-interacting spin one-half systems and by Stevenson [15] for two strongly-interacting spin one-half systems; for a more general discussion the reader is referred to the review by Owen and Harris [25]. The $g$-tensor can be included by plotting the abscissa of the resonances as $g / g^{\prime}$. With this choice, the abscissa is proportional to the applied field and is therefore directly superimposable on experimental spectra. Because the axis is given in terms of $g / g^{\prime}$, any particular value of the abscissa is proportional to the $g$-value for the resonance, $g$. The value, $g$, can be different for the three crystal orientations and in this way one may account for an anisotropic $g$-tensor.

In order to make the data convenient for use with multifrequency EPR data, we plot the ordinate in terms of $\log (h \nu / D)$. Two EPR spectra taken at different frequencies on the same sample will differ vertically by a distance proportional to the logarithm of the ratio of the two frequencies only. The figures in this article provide an illustration of the use of these resonance diagrams. As examples of this type of diagram Figs. 1 and 2 show the principal axis resonances of an $S=2$ and an $S=$ $5 / 2$ spin system. Because of the mathematical form of Eqns. 15, 17 and 18, the Hamiltonian matrices for these operators always block into two submatrices as previously mentioned. The allowed magnetic dipole transitions are always between these submatrices and are proportional to $\mid<S_{+}$ or $S_{-}>\left.\right|^{2}$. In Figs. 1 and 2 , we show all the transitions possible between these submatrices with the restriction that $\left|<S_{+}>\right|^{2}+\left|<S_{-}>\right|^{2}$ is greater than 0.01 . Thus, the abrupt termination of a line such as those near the left-hand margins in Fig. 2 is explained by its 'intensity' having fallen below 0.01. Although, our decision to omit the weak resonances is somewhat simplistic and arbitrary, we do not intend these diagrams to substitute for spectral simulations. They are intended solely to demonstrate lineshift trends from multi-

Fig. 2. Positions of the EPR resonances along the principal axis directions of an $S=5 / 2$ spin system with $\eta=0.07$ : A, magnetic field along the $x$-axis; B, magnetic field along the $y$-axis; $C$, magnetic field along the $z$-axis.
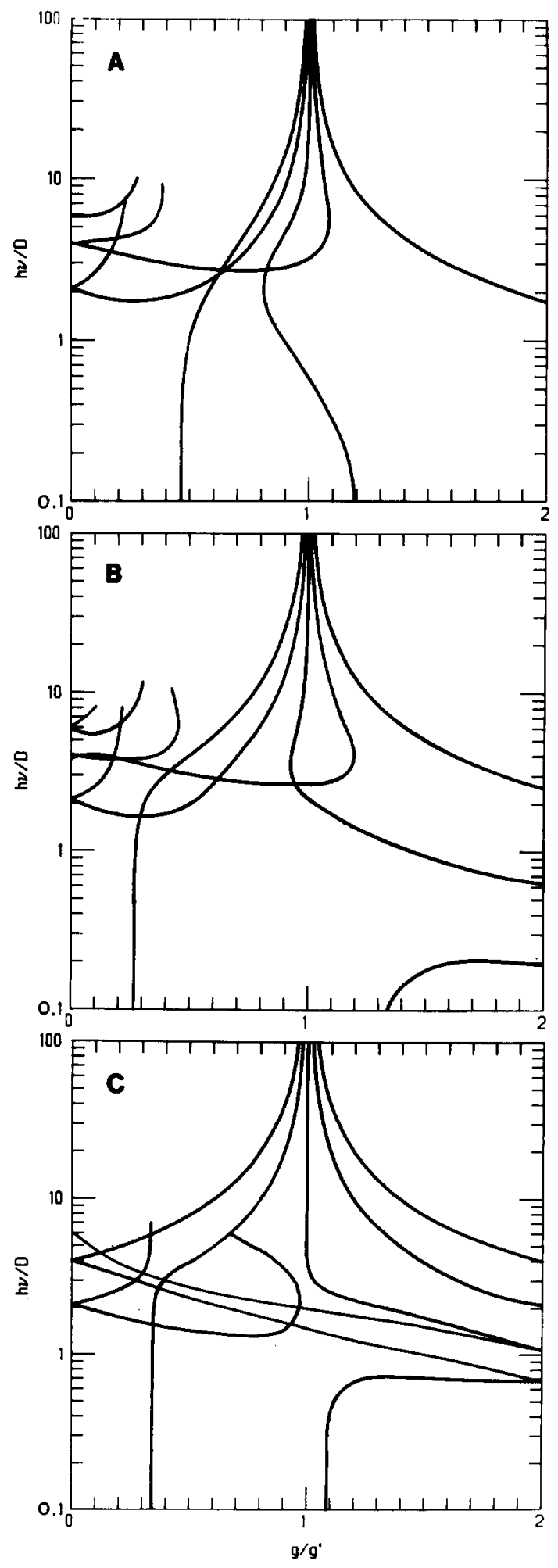
frequency EPR data. From Fig. 2 one can see that if $h \nu / D$ is less than 0.1 , then the resonances are at a constant value $g / g^{\prime}$ so that this system exhibits a microwave frequency-independent spectrum in this region. In contrast, Fig. 1 shows that the integer spin system has no resonances in this region.

\section{Results}

In Fig. 3, we show the X-band spectrum of bovine heart mitochondrial cytochrome $a a_{3}$ which was anaerobically reduced by NADH plus phenazine methosulfate [12], then oxygenated and quickly frozen in an EPR tube. The signals from cytochrome $a$ ( $g$ values: $3.0,2.2,1.5$ ) and $\mathrm{Cu}_{\mathrm{A}}$, not shown, ( $g$ values: $2.18,2.03,1.99$ ) dominate the spectrum. However, we are interested here in the resonances at $g^{\prime}$ equal to $5,1.78$ and 1.70 , which have been shown to appear immediately after reoxygenation and which then disappear simultaneously at room temperature with a half-life of seconds to minutes [26]. This signal will be referred to in the following as the ' $\mathrm{g} 5$ ' signal, although this name is only appropriate to one turning-point of the EPR signal at X-band and not appropriate to any feature of the signal at any other frequency.

If we study the amplitude of these signals versus sample temperature at various microwave powers (Fig. 4), we find that this signal disappears at low temperature, with a characteristic temperature of around $10 \mathrm{~K}$. The points at higher powers and low

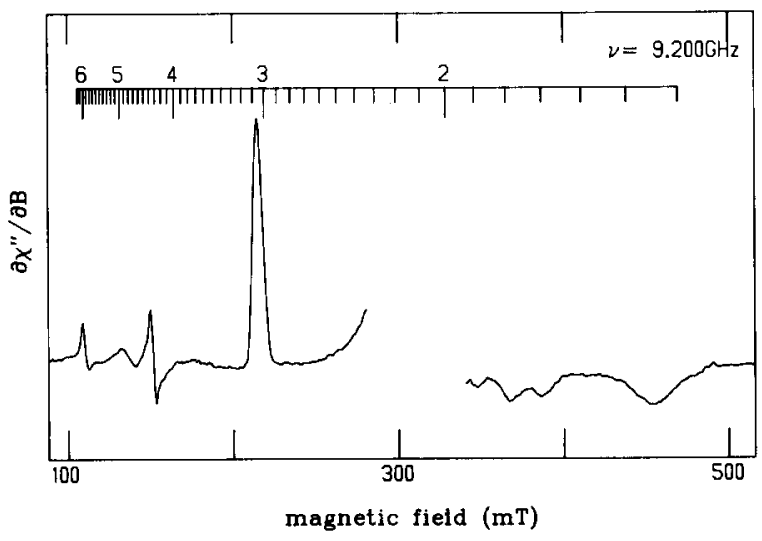

Fig. 3. The $9 \mathrm{GHz}$ EPR spectrum of pulsed cytochrome $a a_{3}$ run at $2.7 \mathrm{~mW}$ and $13 \mathrm{~K}$.

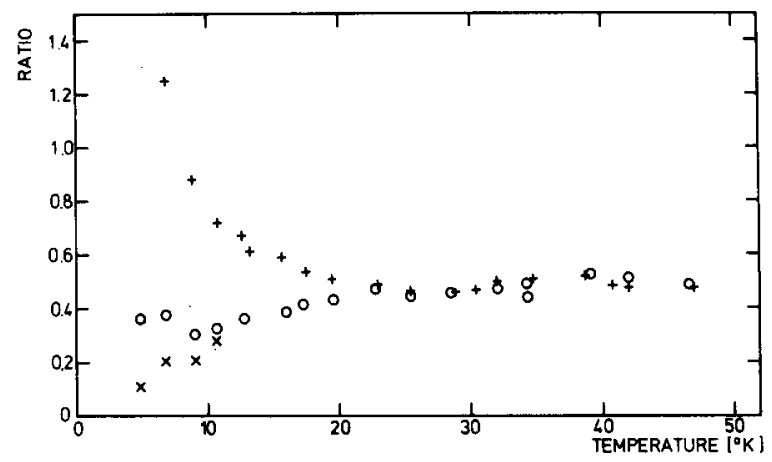

Fig. 4. The temperature and incident microwave power dependece of the ratio of the $g 5$ to cytochrome $a$ X-band EPR signals. The $g 5$ signal was measured at $g=1.70$ and 1.76 ; the cytochrome $a$ signal was measured at $g=1.5$. x, $0.3 \mathrm{~mW} ; O, 3$ $\mathrm{mW}$ +, $30 \mathrm{~mW}$.

temperatures which are above the low power asymptote reflect the saturation of the cytochrome $a$ signal which provided the internal reference for these measurements. Therefore, the other piece of information apparent in these data is that the ' $\mathrm{g} 5$ ' signal is much harder to saturate than the cytochrome $a$ signal.

Figs. 5 and 6 show the 15 and $3 \mathrm{GHz}$ EPR spectra of the same sample as that shown above at $9 \mathrm{GHz}$. At $15 \mathrm{GHz}$ (Fig. 5), we find that the ' $\mathrm{g} 5$ ' signal maxima have all moved toward the $\mathrm{Cu}_{\mathrm{A}}$ signal around $g=2$. These signals show a temperature dependence consistent with that in Fig. 4,

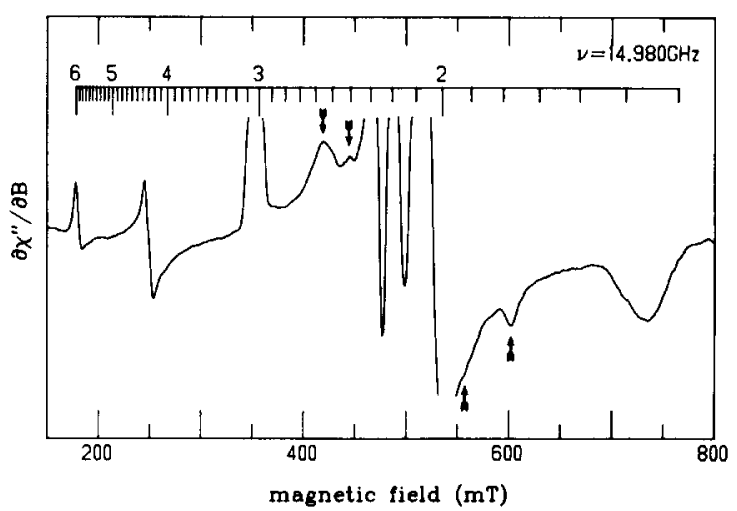

Fig. 5. The $15 \mathrm{GHz}$ EPR spectrum of pulsed cytochrome $a a_{3}$, run at $27 \mathrm{~mW}$ and $30 \mathrm{~K}$. The positions of the ' $\mathrm{g}=5$ ' resonances are shown by arrows. The inner two resonances are more prominent under conditions where $\mathrm{Cu}_{A}$ is saturated. 

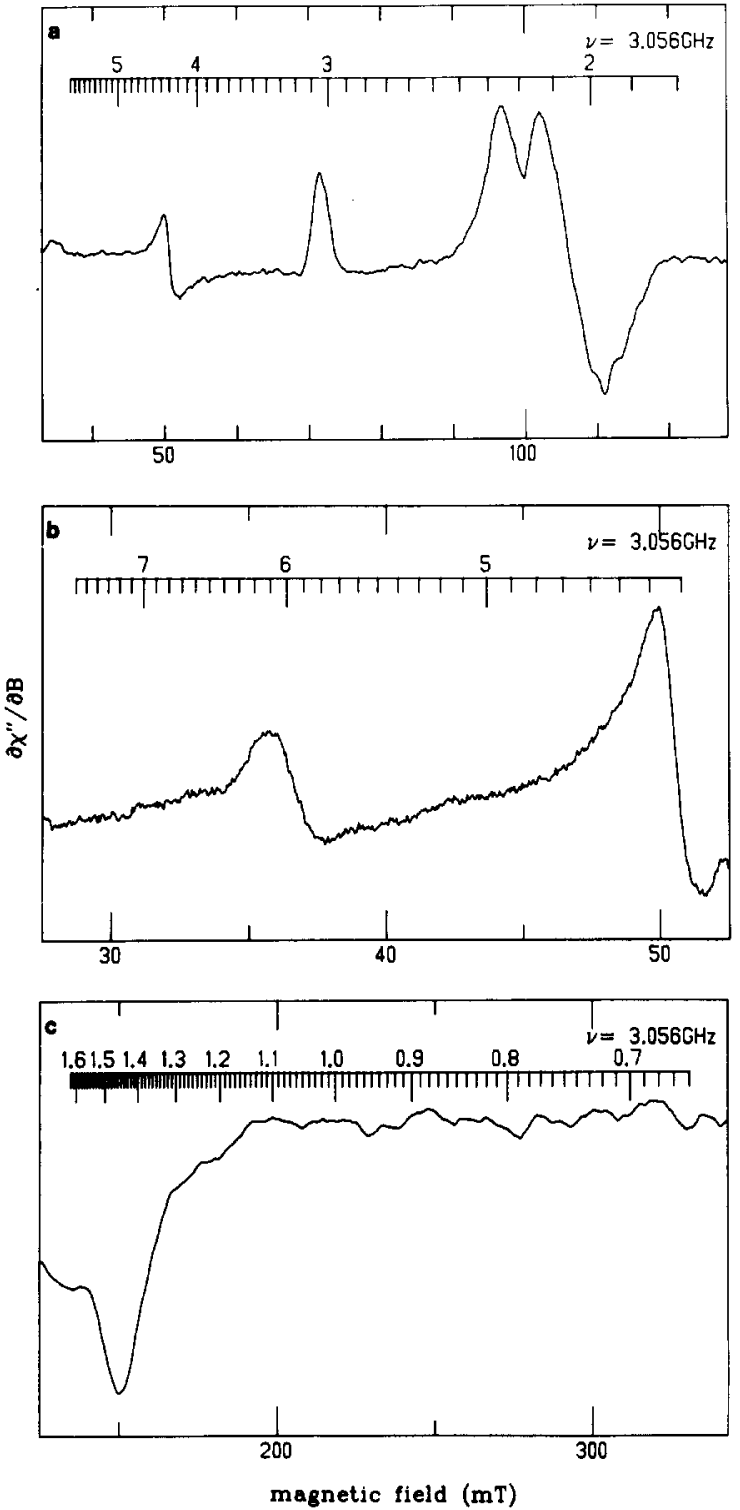

Fig. 6. The $3 \mathrm{GHz}$ EPR spectrum of pulsed cytochrome $a a_{3}$ run at $10 \mathrm{~mW}$ and $10 \mathrm{~K}$ : (a) normal scan range; (b) detail of (a) to show the absence of $g 5$ signal; (c) high-field region to show the absence of resonances predicted for this region (see Fig. 11).

although the diminished signal to noise at $15 \mathrm{GHz}$ has prevented us from showing that the temperature dependence is exactly the same as that seen in the $9 \mathrm{GHz}$ spectra. At $3 \mathrm{GHz}$ we obtained the spectra shown in Fig. 6. These spectra show only the cytochrome $a$ and $\mathrm{Cu}_{\mathrm{A}}$ signals with small additions from 'adventitious' iron at $g=4.3$ and at

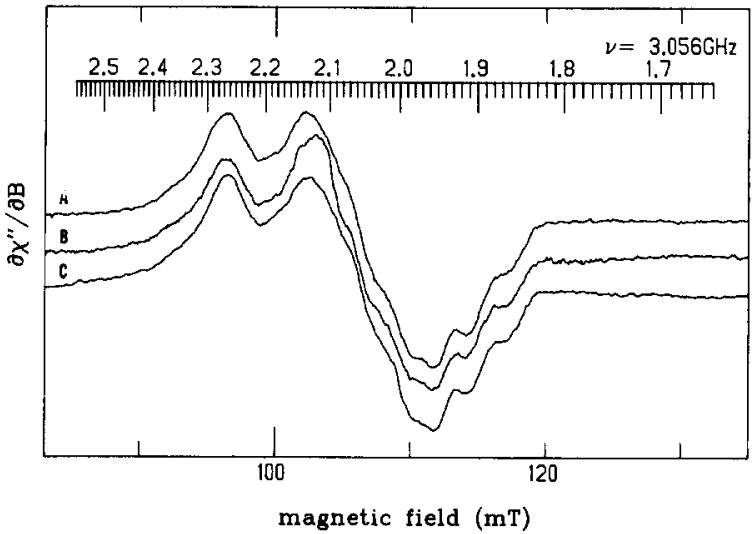

Fig. 7. The $3 \mathrm{GHz}$ EPR spectrum of $\mathrm{Cu}_{\mathrm{A}}$ in three forms of oxidized cytochrome $a a_{3}$, taken at $750 \mathrm{~mW}$ microwave power and at $31 \mathrm{~K}: A$ is the resting enzyme, $B$ is the pulsed enzyme, and $C$ is the anaerobically reoxidized enzyme.

$g=6$ (cytochrome $a_{3}$ ?). One interesting aspect of Fig. 6 is the apparent loss in amplitude of the $\mathrm{CU}_{\mathrm{A}}$ signal relative to the others if one compares Fig. 6 with Figs. 3 or 5 . At $3 \mathrm{GHz}$, magnetic hyperfine interactions broaden the apparent linewidth of the copper so that its intensity seems reduced in comparison with cytochrome $a$. In fact, the magnetic hyperfine splittings are resolved at $3 \mathrm{GHz}$ [27]. In Fig. 7, we show the $3 \mathrm{GHz}$ EPR spectrum of three different forms of cytochrome $a a_{3}$ : the resting

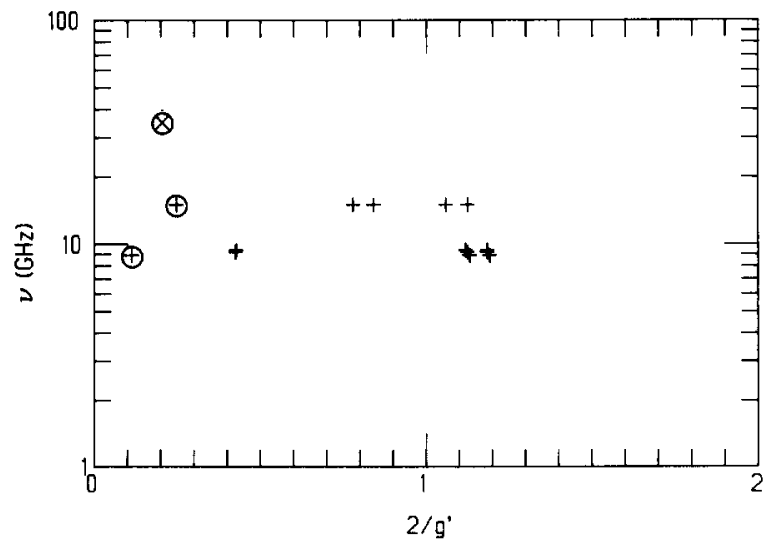

Fig. 8. The positions of the extrema of the 85 signal as a function of microwave frequency. The three left-most points (encircled) are from the $\mathrm{g} 12$ signal of the resting state of cytochrome $a a_{3}$. The point designated by the $\mathrm{X}$ is the $34 \mathrm{GHz}$ point, whose strength is so low that we are uncertain whether to assign it to the $\mathrm{g} 12$ form of the enzyme (see text.) 


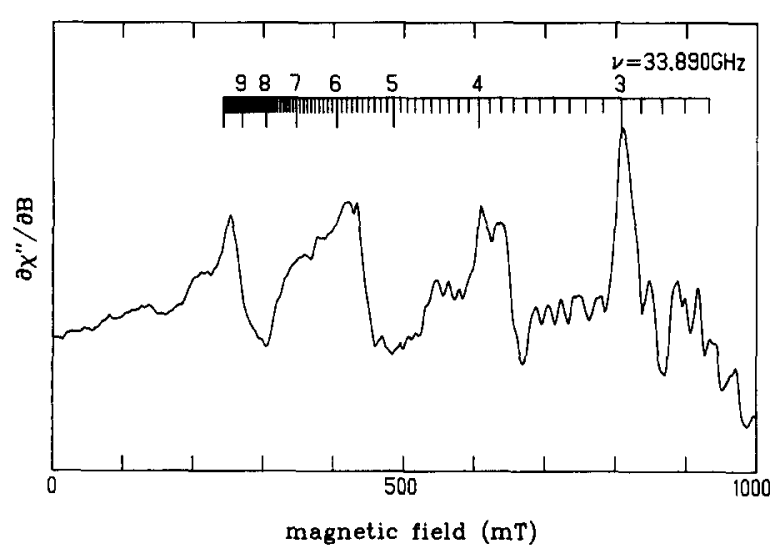

Fig. 9. The $34 \mathrm{GHz}$ EPR spectrum of resting cytochrome $a a_{3}$, run at $0.3 \mathrm{~mW}$ and $17 \mathrm{~K}$.

state, the pulsed state and the anaerobically reoxidized state. These three states of the enzyme are thought to be three different forms of the same oxidized protein. Since the hyperfine splittings in Fig. 7 are the same in all three forms, we have evidence that the differences between these forms, as seen at the $\mathrm{Cu}_{\mathrm{A}}$ atom, are small.

In addition, spectra were taken at 8.9 and 9.4 $\mathrm{GHz}$. We show the results of these experiments in Fig. 8 where the positions of the spectral extrema are plotted as log microwave frequency versus $2 / g^{\prime}$. For the resonances with $g^{\prime}$ less than 2 in Fig. 8 , one can see clearly the trend for the resonances to move closer to the $g=2$ region as the microwave frequency is increased. Fig. 8 also contains

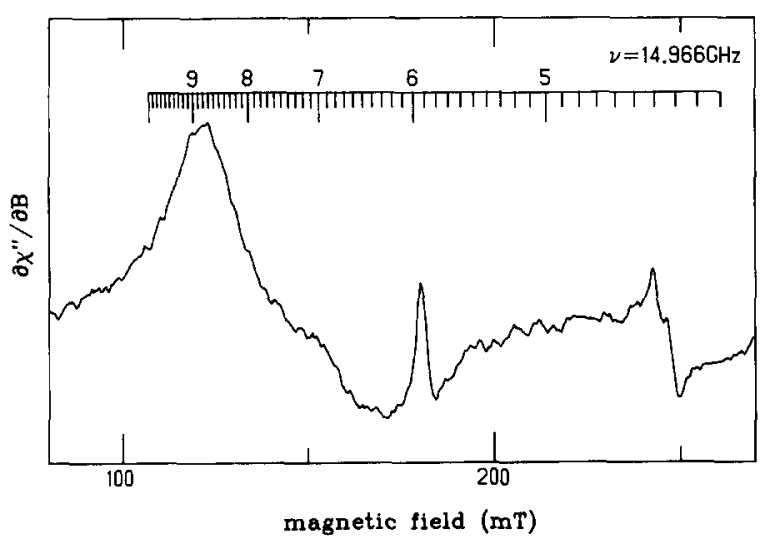

Fig. 10. The $15 \mathrm{GHz}$ EPR spectrum of resting cytochrome $a a_{3}$, run at $27 \mathrm{~mW}$ and $30 \mathrm{~K}$. three points which denote the resonance positions of the ' $g 12$ ' signal of the resting enzyme versus microwave frequency. This resonance is also seen to move closer to the $g=2$ region as the microwave frequency is increased. We show the datum point for the $34 \mathrm{GHz}$ resonance from the resting state as an $\mathrm{X}$ because of the low quality of the spectrum for the point. Fig. 9 shows a typical 34 $\mathrm{GHz}$ spectrum for the resting state of cytochrome $a a_{3}$. For completeness, Fig. 10 shows the $15 \mathrm{GHz}$ spectrum of the 'resting state'.

\section{Discussion}

We have hypothetically identified the extrema in X- and P-band spectra (shown in Fig. 8) with the locations of the resonances along the principal axis directions for the Hamiltonian in Eqns. 15-18. In other words, we identify the resonances with locations denoted in the diagrams, such as those in Figs. 1 and 2. Attempts at this procedure have been equally successful for any spin system with $S=1$ to $S=3$ if we choose $\eta$ to be around 0.07 . In all these cases $D$ is $0.035 \pm 0.015 \mathrm{~cm}^{-1}$. In addition, our attempts [11] to fit these data to similar diagrams which show the resonance positions of an $S=1 / 2$ system with a large magnetic hyperfine coupling to an $I=1 / 2$ nucleus show that this possibility is incompatible with the data. We were also unable to fit these spectra with our 'weak spin-spin interaction' simulation program [24], which handles the cases where the exchange and/ or dipole-dipole interaction is smaller than $g \beta H$ for two interacting spin one-half systems. We are therefore able to propose that $D$ is approximately equal to $0.035 \mathrm{~cm}^{-1}$ for the spin system which gives rise to the resonances in Fig. 8 regardless of the integral value of $S$ for the spin system.

Two main consequences of this statement are that (1) all the states in the spin system are populated thermally at the temperatures appropriate to these data, and (2) the value of $D$ is too small to account for the temperature dependence in the data shown in Fig. 4. While the appreciate that all the transitions which are denoted in the resonance position diagram need to have the same linewidth, if we plot diagrams which show only the transitions to the ground state (Fig. 11), the fitting procedure gives the same value of $D$. However, it 

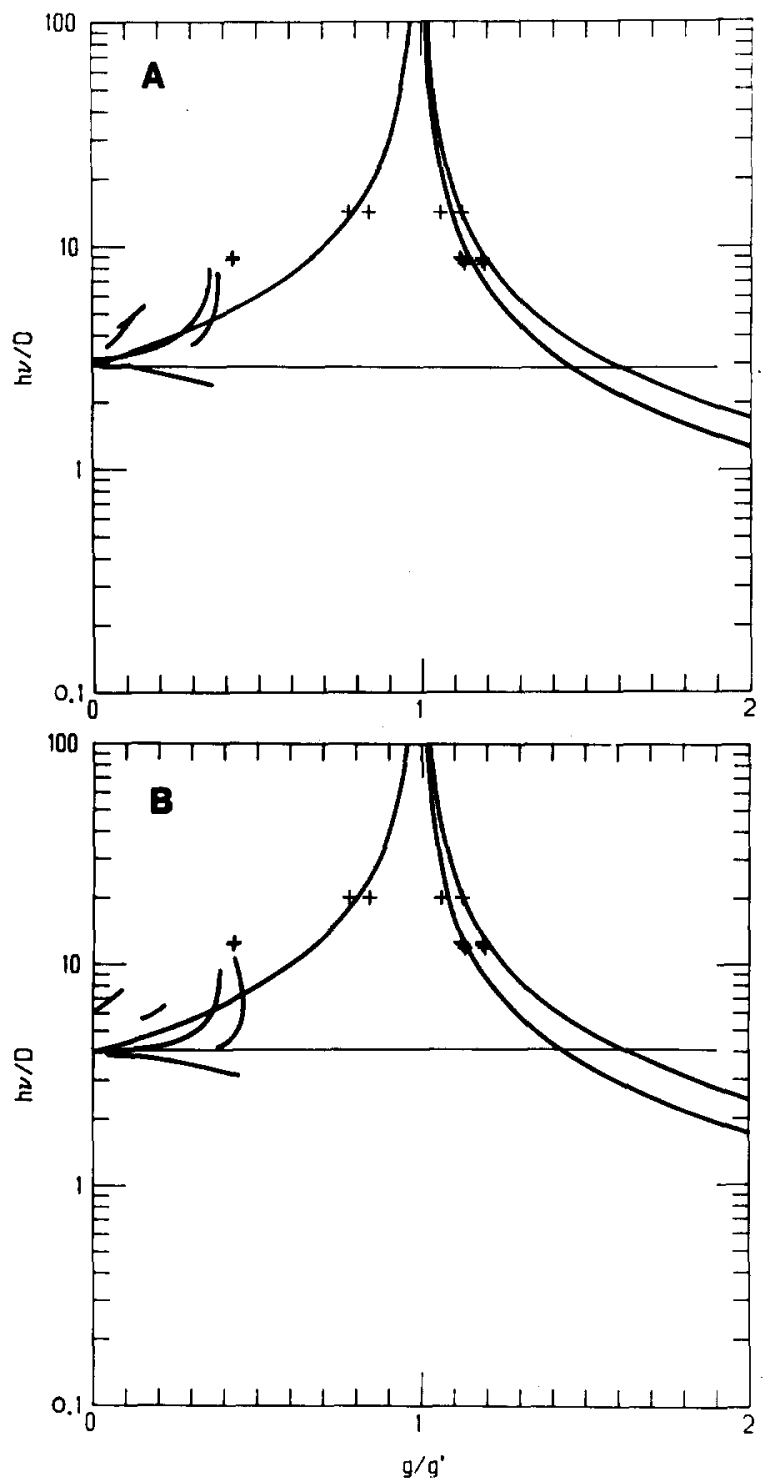

Fig. 11. EPR resonance position diagram for $S=2$ (A) and $S=5 / 2$ (B) spin systems with $\eta=0.07$. In these diagrams only the transitions to the ground state, with $D$ being negative, are shown. The experimental resonance positions of the $g 5$ signal are shown superimposed, with the horizontal line representing the ordinate of the $3 \mathrm{GHz}$ resonances, which were not observed.

is also obvious that the small value of $D$ together with the temperature dependence of the EPR signal imply that there is a lower level of states which is outside the range of frequencies spanned by the EPR-detectable spin system. Thus, the ground state of the detectable spin multiplet is itself an excited state relative to the lower-lying multiplet. The nature of the interaction responsible for creating this energy separation between the excited and ground states cannot be inferred from our data, but will require additional physical measurements. We can only conclude that the EPR signals whose temperature dependence is shown in Fig. 4 arise from an excited manifold and that we are unable to detect any magnetic resonance absorption of the ground state. To state the above arguments in another way, we point out that the only probable sources for the temperature dependence in Fig. 4 are $D$ and $J$. If the $10 \mathrm{~K}$ splitting is from a term in $D$, then the frequency dependence of these signals is from another term. We have eliminated exchange and magnetic hyperfine as possible sources for the $g 5$ signal. High-order terms in $S$ are not considered in this paper, but have been proposed to explain this signal (see Ref. 24 and below). At this time we are unable to rationalize the presence of these resonances with quartic spin terms, but we have efforts underway to simulate these signals. We conclude that our interpretation is a sufficient but not necessary explanation of the data. At present, it is the only explanation of the multifrequency characteristics of the data.

Further corrobotation is provided by the S-band EPR spectrum in Fig. 7. By process of elimination cytochrome $a_{3}$ has been implicated in the spin system under study here. That is, cytochrome $a$ and $\mathrm{Cu}_{\mathrm{A}}$ give rise to the main resonances; $\mathrm{Cu}_{\mathrm{B}}$ can either be in an $S=1 / 2$ or $S=0$ state. Since neither of these states can give rise to the $g 5$ signal, cytochrome $a_{3}$ is the only paramagnetic center in the enzyme left to account for the signal. The spin state of cytochrome $a_{3}$ has been proposed to be either $S=3 / 2$ or $S=5 / 2$ for this state of the enzyme [1]. Examination of Fig. 2 reveals that $S=\$ / 2$, for example, can account for the resonance positions in Fig. 8 by itself. Therefore, one might propose that these signals arise from cytochrome $a_{3}$ alone, while proposing some other explanation for the absence of the $\mathrm{Cu}_{B}$ EPR signal. However, one can see in Fig. 2 that the $\mathrm{X}$-band resonance at $\mathrm{g} 5$ should appear at $\mathrm{S}$-band frequencies in the $g 6-g 4$ region. By extrapolating from the X-band spectra, the intensity of this transition should increase so that it must be seen 
in this region as a line of intensity approximately equal to the $g 6$ signal. Inspection of Fig. 6 reveals that not even the smallest vestige of this signal is present in the data. Similar arguments can eliminate all the half-integer spin systems as candidates for the resonance in Fig. 4. In this context, one can recall from previous discussion that non-integer spin systems give rise to vertical lines on the lower part of the resonance diagrams such as the one in Fig. 2. Inspection of Fig. 1 shows that the S-band spectra should occur at an ordinate where these resonances are in this vertical stage. We can, therefore, reject the non-integer spin systems and thereby require the presence of a coupling to another non-integer spin system in order to account for the absence of a resonance in the $g 4-g 6$ region of the S-band spectrum. The $\mathrm{Cu}_{\mathrm{B}}$ atom is an obvious candidate for this other system. Of course, this requirement is also based on the assumption that cytochrome $a_{3}$ has a ferric iron in this state of the enzyme.

If the cytochrome $a_{3}$ is high-spin $\left(S_{1}=5 / 2\right)$, then the possible resultant spin states are $S=2$ and $S=3$. If, as indicated by MCD results [1], the cytochrome $a_{3}$ is $S_{1}=3 / 2$, then the possible resultants are $S=2$ and $S=1$. In either case, the temperature dependence of the EPR signal (Fig. 4) could be accounted for by an absolute magnitude of $J$ in Eqn. 2 of approximately $1.5 \pm 1 \mathrm{~cm}^{-1}$. In this context, we should also note that the EPR signal under discussion here is harder to saturate than the cytochrome $a$ signal (see Fig. 4).

There are also other mathematical consequences of spin-coupling hypotheses between cytochrome $a_{3}$ and $\mathrm{Cu}_{\mathrm{B}}$ which should be mentioned. For each hypothetical coupling scheme it is possible to write the $g$-value equation for the resultant spin system, to estimate the size of the $\mathrm{Cu}_{B}$ nuclear magnetic hyperfine contribution to the resultant spin and to calculate the size of $D$, the zero-field splitting in the resultant spin system, which should arise from the zero-field splitting, $D_{1}$, at the cytochrome $a_{3}$ iron atom. Table I shows these numbers for the models relevant to the above discussion under the assumptions that the $\mathrm{Cu}_{B}$ site has the non-interacting $g$ values $(2.02-2.36)$ and $a$ values (15-150 gauss) characteristic of the copper site in bovine superoxide dismutase [28]. It is interesting to note from this table that spin coupling alone can account for the absence of visible copper magnetic hyperfine interactions in these EPR spectra. It is also interesting that the measured value of $D$ from our work gives rise to a value of $D_{1}$ which is much smaller than expected for any of the models. Evidently, the iron of cytochrome $a_{3}$, if it is ferric, must be in a state of very high symmetry in this form of the protein as opposed to its form seen during redox titrations [12], where it is undergoing a comparatively strong axial distortion. Because we envision no more at-

TABLE I

SPIN-COUPLING MODELS FOR CYTOCHROME $a a_{3}$

\begin{tabular}{lllllll}
\hline$S_{1}$ (ion) & $S_{2}$ (ion) & $S$ & $g$-value equation & $D$ & $\begin{array}{l}g \text {-value } \\
\text { range }\end{array}$ & $\begin{array}{l}a \text {-value } \\
\text { range }\end{array}$ \\
\hline$\frac{1}{2}\left(\mathrm{Fe}^{3+}\right)$ & $\frac{1}{2}\left(\mathrm{Cu}^{2+}\right)$ & 1 & $g=\frac{1}{2} g_{\mathrm{Fe}}+\frac{1}{2} g_{\mathrm{Cu}}$ & $D=0$ & $?$ & $7.5-75$ \\
$\frac{1}{2}\left(\mathrm{Cu}^{2+}\right)$ & $\frac{1}{2}\left(\mathrm{Cu}^{2+}\right)$ & 1 & $g=\frac{1}{2} g_{\mathrm{Cu}}+\frac{1}{2} g_{\mathrm{Cu}}$ & $D=0$ & $2.01-2.36$ & $15-83$ \\
$\frac{5}{2}\left(\mathrm{Fe}^{3+}\right)$ & $\frac{1}{2}\left(\mathrm{Cu}^{2+}\right)$ & 2 & $g=\frac{7}{6} g_{\mathrm{Fe}}-\frac{1}{6} g_{\mathrm{Cu}}$ & $D=\frac{4}{3} D_{1}$ & $1.94-2.00$ & $2-25$ \\
$\frac{5}{2}\left(\mathrm{Fe}^{3+}\right)$ & $\frac{1}{2}\left(\mathrm{Cu}^{2+}\right)$ & 3 & $g=\frac{5}{6} g_{\mathrm{Fe}}+\frac{1}{6} g_{\mathrm{Cu}}$ & $D=\frac{2}{3} D_{1}$ & $2.00-2.06$ & $2-25$ \\
$2\left(\mathrm{Fe}^{2+}\right)$ & $\frac{1}{2}\left(\mathrm{Cu}^{2+}\right)$ & $\frac{3}{2}$ & $g=\frac{6}{5} g_{\mathrm{Fe}}-\frac{1}{5} g_{\mathrm{Cu}}$ & $D=\frac{7}{5} D_{1}$ & $?$ & $3-30$ \\
$2\left(\mathrm{Fe}^{2+}\right)$ & $\frac{1}{2}\left(\mathrm{Cu}^{2+}\right)$ & $\frac{5}{2}$ & $g=\frac{4}{5} g_{\mathrm{Fe}}+\frac{1}{5} g_{\mathrm{Cu}}$ & $D=\frac{3}{5} D_{1}$ & $?$ & $3-30$ \\
$\frac{3}{2}\left(\mathrm{Fe}^{3+}\right)$ & $\frac{1}{2}\left(\mathrm{Cu}^{2+}\right)$ & 1 & $g=\frac{5}{4} g_{\mathrm{Fe}}-\frac{1}{4} g_{\mathrm{Cu}}$ & $D=\frac{3}{2} D_{1}$ & $?$ & $4-38$ \\
$\frac{3}{2}\left(\mathrm{Fe}^{3+}\right)$ & $\frac{1}{2}\left(\mathrm{Cu}^{2+}\right)$ & 2 & $g=\frac{3}{4} g_{\mathrm{Fe}}+\frac{1}{4} g_{\mathrm{Cu}}$ & $D=\frac{1}{2} D_{1}$ & $?$ & $4-38$ \\
\hline
\end{tabular}


tractive alternative, our interpretation of the EPR signals forces us to predict some major structural change at the cytochrome $a_{3}$ site between the ' $g=5$ ' state of the protein, the half-reduced and the 'rapidly, but anaerobically reoxidized' [29] states of the protein.

If we consider the importance of the absence of EPR from the ground $S$-multiplet we can deduce another fact from the size of $D$. If $D$ is from the exchange or dipole-dipole interactions, then its value is identical for all the multiplets in the spin-coupling scheme. If $D$ is from 'projected zero-field splittings of cytochrome $a_{3}$, then its relative values for the $S$-multiplets are given in Table I. Of all these spin-coupled possibilities, the only one that could explain the absence of an EPR signal in the ground state multiplet is the hypothesis that cytochrome $a_{3}$ is $S=3 / 2$ coupled to copper to give $S=1$ in the ground state and $S=2$ in the excited state. In all other spin-coupled cases, the value of $D$ in the ground state should be such that our efforts to find this ground state would have been successful. However, our direct evidence only allows us to state that the ' $g 5$ ' signal from pulsed cytochrome $a a_{3}$ arises from an excited state with integer spin and $D \simeq 0.035 \mathrm{~cm}^{-1}$ and that this excited state is formed via spin-coupling so that it is approximately $10 \mathrm{~K}$ above the ground state. We are unable to give a precise value for any hypothesized $J$ or to identify the component spin systems, although the information in Table I sets forth a large number of constraints which should be useful in their identification.

In the preceding paragraphs we have described the mathematical framework for calculations involving coupled spin systems. In some recent work, similar calculations have been published by Brudwig et al. [30]. We have several comments to make concerning this work. Without the benefit of the data from multifrequency EPR, this group has attempted an interpretation of the EPR resonances of a spin system which is formed via an exchange interaction. In their interpretation of the ' $\mathrm{g} 12$ ' signal from cytochrome $a a_{3}$ they have attributed this resonance to the spin coupling between cytochrome $a_{3}$ as high-spin ferric and $\mathrm{Cu}_{\mathrm{B}}$ as cupric. While this assignment may be correct, it is not the only possible solution to the problem. By compar- ing the data on the ' $\mathrm{g} 12$ ' resonance in Fig. 4 with diagrams such as those in Figs. 1 and 2, we are able to obtain a sufficient fit with $D \simeq 0.15 \mathrm{~cm}^{-1}$ for any spin system with $S$ greater than one, including $S=5 / 2$ which could arise from the cytochrome $a_{3}$ alone.

In their discussions of the ' $g 5$ ' signal, they assign a solution to the EPR resonances without considering the importance of the 'projected' zero-field splittings or the off-diagonal terms in the $J$-tensor or the nuclear hyperfine coupling to the $\mathrm{Cu}_{\mathrm{B}}$ nucleus. Any explanation of these resonances, in our opinion, requires a discussion of these terms, since one would normally expect them to make large contributions to the spin Hamiltonian. For example, if the cytochrome $a_{3}$ is involved in the coupled spin system, then our EPR work implies that the value of $D_{1}$, the zero-field splitting at cytochrome $a_{3}$, is much smaller than one would expect from a high-spin ferric heme compound. It is very hard to rationalize this size of $D_{1}$ with the structure implied by EXAFS data [31] on the cytochrome $a_{3}$ site.

In another recent paper [32], Hagen has shown data and theory to support the hypothesis of Seiter and Angelos [33] that the resting enzyme contains an $\mathrm{Fe}^{4+}$ site. Hagen proposes that not only is there an $\mathrm{Fe}^{4+}$ in cytochrome $a a_{3}$, but that this iron is in cytochrome $a_{3}$, in a high-spin configuration ( $S=$ 2 ), and gives rise to the $g 12$ and $g^{5}$ signals in reoxidized cytochrome $a a_{3}$ [34]. An $S=2$ configuration is compatible with our data on the $g^{5}$ signal; however, not with a $D$ of $1 \mathrm{~cm}^{-1}$ [32]. An $S=2$ configuration is also compatible with our data on the $g 12$ signal; however, we would not agree with the value of $D$ as $1.2 \mathrm{~cm}^{-1}$ because of the frequency dependence of the $g 12$ signal shown in Fig. 8.

Our purpose is to point out that a solution to these problems demands more data than have hitherto been applied. Our study of these systems included EPR spectra taken at 3, 8.9, 9.2, 9.4, 15 and $34 \mathrm{GHz}$. We were able to garner only a few hard facts from these data, but were able to define the problem more clearly. At this point, we feel that another spectroscopic tool, for example, Mössbauer spectroscopy, is necessary to find a unique solution. 


\section{Acknowledgements}

The authors thank J.A. Fee for allowing us to modify his Varian E-line bridge to make the Xband measurements, R.L. Stevenson for his help with the matrix algebra, W.E. Downer for providing the technical expertise which allowed the successful construction of the S- and P-band cavities, and D.O. Hearshen and H.J. Grande for helping take the data. This work was supported in part by the U.S. Public Health Service via grants GM12176 (R.H.S.) and GM12394 (H.B.), a Research Career Award (5-K06-GM-18442) to H.B. and a National Research Service Award (S-F32-GM-05772) to R.W.S.

\section{References}

1 Carter, K., Antalis, T.M., Palmer, G., Ferris, N.S. and Woodruff, W.H. (1981) Proc. Natl. Acad. Sci. U.S.A. 78, 1652-1655

2 Thompson, A.J., Johnson, M.K., Greenwood, C. and Gooding, P.E. (1981) Biochem. J. 193, 687-697

3 Johnson, M.K., Eglinton, D.G., Gooding, P.E., Greenwood, C. and Thomson A.J. (1981) Biochem. J. 193, 699-708

4 Clore, G.M., Andréasson, L.E., Karlsson, B., Aasa, R. and Malmström, B.G. (1980) Biochem. J. 185, 139-154

5 Clore, G.M. Andréasson, L.E., Karlsson, B., Aasa, R. and Malmström, B.G. (1980) Biochem, J. 185, 155-167

6 Brunori, M., Colosimo, A., Rainoni, G., Wilson, M.T. and Antonini, E. (1979) J. Biol. Chem. 254, 10769-10775

7 Shaw, R.H., Hansen, R.E. and Beinert, H. (1978) J. Biol. Chem. 254, 6637-6640

8 Shaw, R.H., Hansen, R.E. and Beinert, H. (1979) Biochim. Biophys. Acta 548, 386-396

9 Dowsing, R.D. and Gibson, J.F. (1969) J. Chem. Phys. 50, 294-303.

10 Aasa, R. (1970) J. Chem. Phys. 52, 3919-3930.

11 H. Beinert, R.W. Shaw, W.R. Dunham and R.H. Sands (1982) in Oxidases and Related Redox Systems, (King, T.E., Mason, H.S. and Morrison, M., eds.), pp. 1067-1080, Pergamon Press, Ltd., Oxford

12 Hartzell, C.R. and Beinert, H. (1976) Biochim. Biophys. Acta 423, 323-338
13 Beinert, H., Hansen, R.E. and Hartzell, C.R. (1976) Biochim. Biophys. Acta 423, 339-355

14 L.H. Strong (1976) Biophysical Studies on Selected IronSulfur Proteins, Ph.D. thesis, University of Michigan, Ann Arbor, MI

15 R.C. Stevenson (1982) Triplet State EPR: An application To Three Iron-Sulfur Flavoproteins, Ph.D. thesis, University of Michigan, Ann Arbor, MI

16 J.A. Reid (1976) Electron-Nuclear Double Resonance Spectrometry on a High-Potential Iron Protein, Ph.D. thesis, University of Michigan, Ann Arbor, MI

17 D.M. Brink and G.R. Satchler (1962) p. 48, Angular Momentum, Clarendon Press, Oxford

18 D.M. Brink and G.R. Satchler (1962) Angular Momentum, p. 30, Clarendon Press, Oxford

19 Moriya, T. (1960) Phys. Rev. 120, 91-98

20 Dzialoshinski, I. (1958) J. Phys. Chem. Solids 4, 241-255

21 W. Kanzig et al. (1971) Proc. XVI Colloque Ampere, p. 127

22 H. Watanabe (1966) Operator Methods in Ligand Field Theory, p. 147, Prentice-Hall, Inc, Englewood Cliffs, NJ

23 Griffith, J.S. (1971) Mol. Phys. 21, 141-143

24 K.L. Schepler (1975) Electron Spin-Spin Interactions in Three Biological Systems, Ph.D. thesis, University of Michigan, Ann Arbor, MI

25 J. Owen and E.A. Harris (1972) in Pair Spectra and Exchange Interactions in Electron Paramagnetic Resonance, (Geschwind, S., ed.), Ch. 6, Plenum Press, NY

26 Armstrong, F., Shaw, R.W. and Beinert, H. (1983) Biochim. Biophys. Acta 722, 61-71

27 Froncisz, W., Scholes, C.P., Hyde, J.S., Wei, Y., King, T.E., Shaw, R.H. and Beinert, H. (1979) J. Biol. Chem. 254, 7482-7484

28 Lieberman, R.A., Sands, R.H. and Fee, J.A. (1982) J. Biol. Chem 257, 336-344

29 Beinert, H. and Shaw, R.W. (1977) Biochim. Biophys. Acta $462,121-130$

30 Brudwig, G.W., Stevens, T.H., Morse, R.H. and Chan, S.I. (1981) Biochemistry 20, 3912-3921

31 Powers, L., Chance, B., Ching, Y. and Angiolillo, P. (1981) Biophys. J. 34, 465-498

32 Hagen, W.R. (1982) Biochim. Biophys. Acta 708, 82-98

33 Seiter, C.H.A. and Angelos, S.G. (1980) Proc. Natl. Acad. Sci. U.S.A. 77, 1806-1808

34 W.R. Hagen (1982) EPR of Metalloproteins, Ph.D. thesis, University of Amsterdam, Amsterdam 